\title{
VARIAÇÃO DA UMIDADE DO MATERIAL COMBUSTÍVEL FLORESTAL EM FUNÇÃO DE ÍNDICES DE PERIGO DE INCÊNDIOS
}

\author{
José Fernando Pereira ${ }^{1}$, Antonio Carlos Batista ${ }^{2}$, Ronaldo Viana Soares ${ }^{3}$
}

(recebido: 29 de abril de 2010; aceito: 28 de fevereiro de 2012)

RESUMO: Esta pesquisa foi realizada em plantios de Pinus elliotii localizados na Estação Florestal Experimental de Rio NegroParaná, pertencente à Universidade Federal do Paraná com os objetivos de analisar as correlações entre a umidade dos combustíveis florestais finos e os índices de perigo de incêndios FMA e FMA+ e desenvolver modelos para estimar a umidade dos materiais combustíveis baseados nesses índices. As variáveis meteorológicas utilizadas na pesquisa foram obtidas junto à estação meteorológica do Instituto SIMEPAR e de um pluviógrafo e de um termohigrógrafo instalados na área do experimento. Os combustíveis florestais foram coletados em parcelas de $30 \mathrm{~cm}$ x $30 \mathrm{~cm}$, classificados por diâmetro $(<0,7 \mathrm{~cm} \varnothing-\mathrm{A}$ e de 0,7 a $2,5 \mathrm{~cm} \varnothing-\mathrm{B})$ e em função da posição na manta (camada superficial - AA, intermediária - AB e inferior - AC). A espessura da manta formada pelos combustíveis acamados sobre o piso florestal variou entre 14,8 e $15,3 \mathrm{~cm}$. A quantidade total de material combustível variou entre 3185,50 e 4266,01 g.m $\mathrm{m}^{-2}$. Os índices de perigo de incêndios FMA e FMA+ foram calculados diariamente e os valores obtidos nos dias das coletas dos combustíveis foram usados para as análises de correlações e ajustes das funções estimativas. Os coeficientes de correlação entre umidade relativa e classes de combustível foram 0,$42 ; 0,36 ; 0,32$ e 0,41 para o AA, $\mathrm{AB}, \mathrm{AC}$, e as classes $\mathrm{B}$, respectivamente. Os coeficientes de correlação entre a precipitação e as classes de combustível foram 0,$57 ; 0,38 ; 0,34$; e 0,15 para o AA, AB, AC e as classes $\mathrm{B}$, respectivamente. Os coeficientes de correlação entre as classes de combustível eo + FMA foram - $-0,53$; $-0,56$; - 0,63 ; e 0,81 para o $\mathrm{B}$ classes, $\mathrm{AB}, \mathrm{AA}$, e AC, respectivamente. $\mathrm{O}$ + FMA foi a variável mais eficiente no desenvolvimento de modelagem para estimar a umidade de combustível morto. A umidade dos materiais combustíveis finos apresentou correlações estatisticamente significativas com as variáveis umidade relativa do ar e precipitação e com os índices de perigo de incêndios FMA e FMA ${ }^{+}$. No desenvolvimento dos modelos para a estimativa de umidade dos materiais a variável mais importante observada nos ajustes foi o FMA ${ }^{+}$.

Palavras-chave: Fórmula de Monte Alegre, Fórmula de Monte Alegre alterada, prevenção de incêndios florestais.

\section{FOREST FUEL MOISTURE VARIATION IN TERMS OF FOREST FIRE DANGER INDEX}

\begin{abstract}
This research was carried out in a Pinus elliottii plantation, established in 1984, located in the Rio Negro Forest Research Station- Rio Negro - Paraná, owned by the Paraná Federal University, Paraná State, Brazil. The research objectives were to analyze the correlations between the FMA and $F M A^{+}$fire danger indices and the fine fuel moisture, and develop mathematical models to estimate the fuel moisture based on these indices. The meteorological variables were obtained from a SIMEPAR weather station, located $50 \mathrm{~km}$ away, and from a pluviograph and a thermo-hygrograph installed in the study area. The dead forest fuels were collected from $30 \times 30 \mathrm{~cm}$ plots, between 12 noon and 2:00PM, and classified as: AA - surface layer; AB - intermediate layer; $A C$-lower layer; and B -woody material with 0.7 to $2.5 \mathrm{~cm}$ diameter. The total fuel load varied from 3185.50 to $4266.01 \mathrm{~g} . \mathrm{m}^{-2}$. The fire danger indices were daily calculated and the values obtained in the fuel colleting days were used to calculate the correlations. The correlation coefficients between relative humidity and fuel classes were 0.42; 0.36; 0.32; and 0.41 for the $A A, A B, A C$, and $B$ classes, respectively. The correlation coefficients between precipitation and fuel classes were 0.57; $0.38 ; 0.34 ;$ and 0.15 for the AA, $A B, A C$, and $B$ classes, respectively. The correlation coefficients between the fuel classes and the FMA $A^{+}$were $-0.53 ;-0.56$; -0.63 ; and 0.81 for the classes $B, A B, A A$, and $A C$, respectively. The $F M A^{+}$was the most efficient variable in modeling development to estimate dead forest fuel moisture.
\end{abstract}

Key words: Monte Alegre Formula, Modified Monte Alegre Formula, Forest Fires prevention.

\section{INTRODUÇÃO}

As áreas reflorestadas com Pinus sp representam, aproximadamente, $34,6 \%$ dos reflorestamentos brasileiros e o estado do Paraná, com uma participação de $37 \%$ do total, ocupa o primeiro lugar em relação à área plantada com pinus no Brasil (NUNES, 2005). A crescente expansão dessas áreas de reflorestamento, principalmente no sul do país, vem provocando uma necessidade contínua de aprimoramento das técnicas de manejo e proteção.

\footnotetext{
${ }^{1}$ Engenheiro Florestal, Professor Doutorando em Engenharia Florestal - Centro Estadual Florestal de Educação Profissional Presidente Costa e Silva Av. Paraná s/no , Bairro Vila São João - 84.500-000 - Irati, PR, Brasil - ultimojose@yahoo.com.br

${ }^{2}$ Engenheiro Florestal, Professor Doutor em Engenharia Florestal - Universidade Federal do Paraná - Setor de Ciências Agrárias - Departamento de Ciências Florestais - Av Lothário Meissner, 900 - 80210-170 - Curitiba, PR, Brasil - batistaufpr@ufpr.br

${ }^{3}$ Engenheiro Florestal, Professor Ph.D. em Engenharia Florestal - Universidade Federal do Paraná - Setor de Ciências Agrárias - Departamento de Ciências Florestais - Av Lothário Meissner, 900 - 80210-170 - Curitiba, PR, Brasil-rvsoares@ufpr.br
}

Cerne, Lavras, v. 18, n. 3, p. 371-376, jul./set. 2012 
Os incêndios florestais constituem-se numa preocupação constante e representam um dos principais objetivos dos planos de proteção para as áreas de reflorestamentos.

A utilização de um índice de perigo de incêndios florestais confiável é fator fundamental para um planejamento mais eficiente das medidas de prevenção e para a adoção de ações rápidas e efetivas nas atividades de combate aos incêndios florestais, visando à redução das perdas e, consequentemente, dos prejuízos financeiros e ambientais advindos da ocorrência de eventos catastróficos. Em virtude da sua importância e da facilidade com que esses índices podem ser calculados atualmente, mediante softwares específicos (NUNES, 2007), é crescente a sua difusão e utilização por entidades ligadas à prevenção e combate aos incêndios florestais no Brasil.

A Fórmula de Monte Alegre (FMA) foi o primeiro índice de perigo de incêndios desenvolvido no Brasil por Soares (1972), sendo empregada largamente em todas as regiões brasileiras e outras regiões do mundo, como América Latina e África, apresentando eficiente desempenho na previsão dos incêndios florestais. Recentemente, o índice FMA foi aperfeiçoado por Nunes (2005), introduzindo-se a variável meteorológica velocidade do vento no modelo tradicional, passando a se denominar Fórmula de Monte Alegre Alterada $\left(\mathrm{FMA}^{+}\right)$, melhorando ainda mais o desempenho do índice na previsão da ignição e propagação dos incêndios.

O conhecimento da umidade dos materiais combustíveis é imprescindível para a estimativa de parâmetros ligados ao comportamento do fogo, tais como: intensidade e velocidade de propagação, além de ser fator decisivo na obtenção de bons resultados com a queima controlada. É também um dos mais importantes fatores a serem analisados para a estimativa do risco de incêndios florestais (SOARES et al., 2009; YEBRA et al., 2008).

No Brasil, a estimativa da umidade do material combustível tem sido feita por meio de medições diretas em amostragem de campo e determinação em laboratório, utilizando-se, normalmente, a pesagem no campo (massa úmida) com posterior secagem até peso constante, quando são novamente pesadas, determinando-se, assim, o teor de umidade da amostra avaliada (BATISTA, 1995).

Conforme Alves et al. (2009), o desenvolvimento de métodos indiretos na determinação da umidade do material combustível poderia agilizar os trabalhos dos técnicos responsáveis pela proteção dessas áreas florestais.

Cerne, Lavras, v. 18, n. 3, p. 371-376, jul./set. 2012
A hipótese de haver correlação entre os índices de perigo de incêndios e a umidade do material combustível pode indicar mais uma importante contribuição dos índices FMA e FMA $^{+}$na prevenção dos incêndios florestais.

Neste trabalho, objetivou-se analisar as correlações entre os índices de perigo incêndios FMA e $\mathrm{FMA}^{+} \mathrm{e}$ a umidade dos combustíveis florestais finos e desenvolver modelos para estimar a umidade dos combustíveis baseados nesses índices.

\section{MATERIAL E MÉTODOS}

\subsection{Localização}

O experimento foi desenvolvido na Estação Experimental de Rio Negro, administrada pelo Curso de Engenharia Florestal da Universidade Federal do Paraná e localizada às margens da rodovia BR-116 ( $\mathrm{km} \mathrm{200),}$ aproximadamente $110 \mathrm{~km}$ de Curitiba (coordenadas $26^{\circ} 04^{\prime} 02,40^{\prime \prime} \mathrm{S}$ e $49^{\circ} 45^{\prime} 58,76^{\prime \prime} \mathrm{W}$ ). A altitude média da região é de 793 metros. A precipitação total média anual é de $1400 \mathrm{~mm}$ e o clima é do tipo $\mathrm{Cfb}$, conforme classificação de Koppen, apresentando temperatura média do mês mais quente inferior a $22^{\circ} \mathrm{C}$, sem estação seca (o mês mais seco apresenta precipitação superior a $60 \mathrm{~mm}$ ) e com mais de 10 geadas por ano.

\subsection{Dados meteorológicos e índices de perigo de incêndios}

De acordo com Soares e Batista (2007), as principais variáveis que controlam a umidade do material combustível são a precipitação, a umidade relativa e a temperatura do ar. $\mathrm{O}$ vento e a radiação solar são fatores importantes na secagem, mas exercem sua influência pela modificação da temperatura dos combustíveis e da temperatura e umidade relativa no fino filme de ar adjacente.

Os dados meteorológicos utilizados neste estudo foram obtidos de uma estação meteorológica pertencente à rede meteorológica oficial do estado do Paraná (Instituto SIMEPAR), localizada aproximadamente a $50 \mathrm{~km}$ da Estação Experimental, e também de um pluviógrafo modelo Hellmann-Fuess e de um termohigrógrafo modelo 8145 Lufft instalados na área do experimento.

Os índices de perigo de incêndios FMA e $\mathrm{FMA}^{+}$ foram calculados diariamente para os anos de 2007 e 2008, por meio de metodologia proposta por Nunes (2005) e Soares (1972) e processados por meio de um programa em linguagem Pascal, desenvolvido por Nunes (2005), utilizando-se das seguintes equações: 


$$
\mathrm{FMA}=\sum_{\mathrm{i}=1}^{\mathrm{n}}\left(\frac{100}{\mathrm{H}_{\mathrm{i}}}\right)
$$

sendo:

FMA = Fórmula de Monte Alegre;

$\mathrm{H}_{\mathrm{i}}=$ Umidade Relativa às 13:00 horas;

$\mathrm{N}=$ número de dias sem chuva.

$$
\mathrm{FMA}^{+}=\sum_{\mathrm{i}=1}^{\mathrm{n}}\left(\frac{100}{\mathrm{H}_{\mathrm{i}}}\right) * \mathrm{e}^{0,04 \mathrm{v}}
$$

sendo:

$\mathrm{FMA}^{+}=$Fórmula de Monte Alegre alterada;

$\mathrm{H}_{\mathrm{i}}=$ Umidade Relativa às 13 horas;

$\mathrm{v}=$ velocidade do vento $\mathrm{em} \mathrm{m} \cdot \mathrm{s}^{-1}$ às 13 horas;

$\mathrm{N}=$ número de dias sem chuva.

Para as análises de correlação normal e elaboração dos modelos para estimativa da umidade do material combustível, os índices foram incluídos na forma dos valores diários calculados e na forma de uma escala de graus de perigo, conforme apresentado na Tabela 1. Esses valores numéricos referem-se ao grau relativo do perigo de incêndios, de acordo com a escala de perigo proposta originalmente por Soares (1972) na metodologia de cálculo do FMA.

Tabela 1 - Graus de Perigo de incêndios dos índices FMA e $\mathrm{FMA}^{+}$, usados nas análises de correlação e modelos matemáticos para estimativa de umidade do material combustível.

Table 1-Degrees of FMA and FMA ${ }^{+}$fire danger indices used in correlation analysis and mathematical models used to estimate fuel moisture content.

\begin{tabular}{lc}
\hline Grau de Perigo & Valor numérico do Grau \\
\hline Nulo & 1 \\
Pequeno & 2 \\
Médio & 3 \\
Alto & 4 \\
Muito alto & 5 \\
\hline
\end{tabular}

\subsection{Amostragem e análise estatística}

A determinação do local para a instalação do experimento foi feita após incursões no talhão, buscando um local representativo das condições médias do povoamento, evitando as bordaduras.

A fase de coleta de campo foi programada para ser desenvolvida durante um ano, contemplando coletas ao longo das quatro estações, com início das coletas no inverno de 2007 e término no outono de 2008, realizandose as coletas nos períodos em que o ambiente estivesse mais seco em cada estação.

O experimento compreendeu um total de 60 dias de coleta, sendo 15 dias por estação do ano e 8 amostras coletadas por dia, perfazendo um total de 480 amostras. Cada unidade de amostra era composta por uma área de 30 x $30 \mathrm{~cm}$. O material combustível foi agrupado em classes diamétricas, de acordo com Brown et al. (1982), com o auxílio de um medidor de diâmetros.

As amostras compreenderam duas classes de material combustível, sendo a classe A, com diâmetro entre 0 e $0,7 \mathrm{~cm}$ e a classe B com diâmetro entre 0,71 a $2,5 \mathrm{~cm}$. O material com diâmetro superior a $2,5 \mathrm{~cm}$ não foi amostrado por apresentar uma amplitude muito grande de umidade e também pelas características relacionadas com o tempo de resposta do material em relação às condições meteorológicas que indicam que combustíveis florestais dessa classe demoram muito tempo para variar o seu conteúdo de umidade.

Em virtude do material combustível depositado no piso do povoamento formar uma manta uniforme, caracterizada por camadas distintas, de acordo com o tempo de decomposição, a classe A foi dividida em três subclasses:

- Camada superior AA - formada por acículas e ramos entre 0 e $0,7 \mathrm{~cm}$ de diâmetro, caracterizada por acículas bastante rígidas, de cor marrom clara brilhante, recentemente caídas;

- Camada intermediária AB - formada por acículas e ramos entre 0 e $0,7 \mathrm{~cm}$ de diâmetro, composta por acículas de coloração marrom, mas com ausência de brilho, com menor rigidez, já apresentando indícios de decomposição;

- Camada inferior AC - formada por acículas e ramos entre 0 e $0,7 \mathrm{~cm}$ de diâmetro, caracterizada por apresentar um material de coloração marrom escuro, em processo de decomposição avançado e baixa rigidez.

$\mathrm{O}$ material com diâmetro entre 0,71 e $2,5 \mathrm{~cm} \mathrm{~B}$ foi caracterizado por conter basicamente ramos, em diferentes estágios de decomposição, presentes em todas as subclasses descritas anteriormente.

No laboratório, o material coletado foi transferido para embalagens de papel Kraft e, em seguida, posto em estufa para secagem a $75^{\circ} \mathrm{C}$ por 72 horas. Após a secagem, determinou-se o teor de umidade, por meio da seguinte equação:

Cerne, Lavras, v. 18, n. 3, p. 371-376, jul./set. 2012 


$$
\mathrm{UMC}=\left(\frac{\mathrm{PU}-\mathrm{PS}}{\mathrm{PS}}\right) * 100
$$

onde:

$\mathrm{UMC}=$ porcentagem de umidade do material combustível; $\mathrm{PU}=$ peso do material úmido (no momento da coleta); $\mathrm{PS}=$ peso do material seco (após a secagem em estufa).

A análise de correlação e a geração dos modelos matemáticos para a estimativa da umidade das classes de material combustível foi realizada por meio do software estatístico STATGRAPHICS PLUS 4.1. Os modelos foram criados por meio do processo backward, que consiste em usar todas as variáveis selecionadas inicialmente. Sequencialmente, as variáveis de menor importância foram retiradas. Para selecionar os melhores modelos e testar a qualidade dos ajustes dos modelos, foram utilizados dois parâmetros de comparação:

a) Coeficiente de Determinação $\left(\mathrm{R}^{2}\right)$ - parâmetro que expressa o quanto as variações da variável dependente são explicadas pelas variáveis independentes.

b) Erro Padrão da Estimativa (Syx) - que expressa o quanto, em termos médios, os valores observados variam em relação aos valores estimados.

Os modelos para estimar a umidade dos combustíveis foram desenvolvidos em função das variáveis meteorológicas (umidade relativa do ar, precipitação e velocidade do vento) e dos índices de perigo de incêndios (FMA e FMA ${ }^{+}$).

Para a interpretação das variáveis descritas nos modelos matemáticos de estimativa da umidade dos materiais combustíveis e nas matrizes de correlação, adotaram-se as convenções descritas na Tabela 2.
Tabela 2 - Variáveis utilizadas na matriz de correlação e na composição dos modelos matemáticos.

Table 2 - Variables used in the correlation matrix and composition of mathematical models.

\begin{tabular}{|c|c|c|}
\hline $\begin{array}{l}\text { Nome da } \\
\text { Variável }\end{array}$ & Descrição & Unidade \\
\hline$E$ & Estações do ano & 1 a 4 \\
\hline $\mathrm{U}_{\mathrm{AA}}$ & $\begin{array}{l}\text { Umidade do material combustível } \\
\text { Superficial (AA) }\end{array}$ & $\%$ \\
\hline $\mathrm{U}_{\mathrm{AB}}$ & $\begin{array}{l}\text { Umidade do material combustível } \\
\text { intermediário }(\mathrm{AB})\end{array}$ & $\%$ \\
\hline $\mathrm{U}_{\mathrm{AC}}$ & $\begin{array}{l}\text { Umidade do material combustível } \\
\text { Inferior (AC) }\end{array}$ & $\%$ \\
\hline $\mathrm{U}_{\mathrm{B}}$ & $\begin{array}{l}\text { Umidade do material combustível da } \\
\text { classe (B) }\end{array}$ & $\%$ \\
\hline $\mathrm{U}$ & Umidade Relativa do ar das 13 horas & $\%$ \\
\hline $\mathrm{P}$ & Precipitação pluviométrica & $\mathrm{mm}$ \\
\hline $\mathrm{V}$ & Velocidade do Vento & $\mathrm{m} \cdot \mathrm{s}^{-1}$ \\
\hline GF & $\begin{array}{l}\text { Grau de perigo - Fórmula de Monte } \\
\text { Alegre }\end{array}$ & 1 a 5 \\
\hline $\mathrm{F}+$ & Fórmula de Monte Alegre Alterada & Valor \\
\hline $\mathrm{GF}+$ & $\begin{array}{l}\text { Grau de perigo - Fórmula de Monte } \\
\text { Alegre Alterada }\end{array}$ & 1 a 5 \\
\hline
\end{tabular}

\section{RESULTADOS E DISCUSSÃO}

A matriz de correlação apresentada na Tabela 3 mostra as correlações existentes entre os índices de perigo de incêndios FMA e $\mathrm{FMA}^{+}$e a umidade das classes dos materiais combustíveis.

Tabela 3 - Correlações entre umidade do material combustível, variáveis meteorológicas e os índices FMA e FMA ${ }^{+}$.

Table 3 -Correlations between the fuel moisture, weather variables and the FMA and FMA ${ }^{+}$indices.

\begin{tabular}{|c|c|c|c|c|c|c|c|c|c|c|}
\hline & $\mathrm{U}_{\mathrm{AA}}$ & $\mathrm{U}_{\mathrm{AB}}$ & $\mathrm{U}_{\mathrm{AC}}$ & $\mathrm{U}_{\mathrm{B}}$ & $\mathrm{U}$ & $\mathrm{P}$ & V & $\mathrm{F}$ & GF & $\mathrm{F}+$ \\
\hline $\mathrm{U}_{\mathrm{AB}}$ & $0,67 * *$ & & & & & & & & & \\
\hline $\mathrm{U}_{\mathrm{AC}}$ & $0,57 * *$ & $0,59 * *$ & & & & & & & & \\
\hline $\mathrm{U}_{\mathrm{B}}$ & $0,61^{* *}$ & $0,68^{* *}$ & $0,65^{* *}$ & & & & & & & \\
\hline $\mathrm{U}$ & $0,42 *$ & $0,36^{*}$ & $0,32 *$ & $0,41 *$ & & & & & & \\
\hline $\mathrm{P}$ & $0,57 * *$ & $0,38^{*}$ & $0,34 *$ & 0,15 & 0,19 & & & & & \\
\hline V & 0,04 & 0,16 & $-0,19$ & $-0,06$ & $-0,17$ & 0,28 & & & & \\
\hline $\mathrm{F}$ & $-0,46^{* *}$ & $-0,46^{* *}$ & $-0,80 * *$ & $-0,49 * *$ & $-0,34 * *$ & $-0,34 *$ & 0,28 & & & \\
\hline GF & $-0,60$ ** & $-0,47 * *$ & $-0,71^{* *}$ & $-0,36^{*}$ & $-0,25$ & $-0,71^{* *}$ & 0,05 & $0,74 * *$ & & \\
\hline $\mathrm{F}+$ & $-0,46^{* *}$ & $-0,46^{* *}$ & $-0,80 * *$ & $-0,49 * *$ & $-0,34^{*}$ & $-0,34^{*}$ & 0,28 & 1,00 & $0,74 * *$ & \\
\hline GF+ & $-0,63 * *$ & $-0,56^{* *}$ & $-0,81^{* *}$ & $-0,53 * *$ & $-0,32 *$ & $-0,57 * *$ & 0,12 & $0,74 * *$ & $0,93 * *$ & $0,86^{* *}$ \\
\hline
\end{tabular}

Cerne, Lavras, v. 18, n. 3, p. 371-376, jul./set. 2012 
O uso da escala de 1 a 5 (graus de perigo do índice) para os cálculos de correlação apresentaram melhores resultados se comparados com os valores diários absolutos do índice. $\mathrm{O}$ índice de perigo $\mathrm{FMA}^{+}$apresentou melhor correlação com a umidade do material combustível quando comparado com o índice FMA, demonstrando que a inclusão da variável velocidade do vento na composição do FMA melhorou a correlação com o material combustível. Dessa forma, para o $\mathrm{GF}^{+}$, os coeficientes de correlação (R) indicaram uma associação de $-0,63 ;-0,56 ;-0,81 ;-0,53$ para as classes AA; AB; AC; e B, respectivamente. Já, para o $\mathrm{F}$, foram obtidas as correlações $-0,60 ;-0,47 ;-0,71 ;-0,36$. As correlações negativas indicam que quanto maior o grau de perigo menor a umidade do combustível. A classe AC apresentou alta correlação com $\mathrm{GF}^{+}(-0,81)$, provavelmente por sofrer alterações menos bruscas de perda ou ganho de umidade, em decorrência de sua posição em relação à manta de combustível.

De maneira geral, a classe B apresentou a menor associação com o $\mathrm{FMA}^{+}$. Esse resultado está associado ao fato de que combustíveis grossos, geralmente lenhosos, reagem mais lentamente às variações de umidade do ambiente (FOSBERG et al., 1970).

A correlação dos índices de perigo de incêndios FMA e $\mathrm{FMA}^{+}$com a umidade do material combustível apresentou melhores resultados quando comparada com a correlação obtida com as variáveis meteorológicas umidade relativa do ar $(\mathrm{U})$, precipitação $(\mathrm{P})$ e velocidade do vento (v) (Tabela 3).

Para o inverno, a melhor estimativa foi obtida para a classe AA $\left(R^{2}=0,59\right)$, por meio de uma única variável $(\mathrm{GF}+)$, como pode se observar na Tabela 4. Para a classe de material B, obteve-se uma fraca estimativa $\left(R^{2}=0,28\right)$, por meio da variável U. Para as classes de combustível AB e AC, nenhum dos modelos testados forneceu estimativas confiáveis de umidade do combustível.

Para a primavera, as melhores estimativas ocorreram para as classes de material $\mathrm{AB}$ e $\mathrm{AC}$, com $\mathrm{R}^{2}$ de 0,76 e 0,79 respectivamente, sendo selecionadas as variáveis $\mathrm{U}_{\text {e }} \mathrm{F}^{+}$ para compor os modelos das duas classes. As melhores estimativas de umidade para a classe AA foram obtidas com as variáveis $\mathrm{U}$ e $\mathrm{P}$. Para a classe $\mathrm{B}$, a melhor estimativa foi obtida com a variável GF, também com um fraco desempenho $\left(\mathrm{R}^{2}=0,33\right)$, como no modelo do inverno.

Para o verão, os modelos para a estimativa de umidade de todas as classes de material apresentaram bom desempenho, com coeficientes de determinação entre 0,6 e 0,85 . É interessante observar que a variável GF+ foi selecionada para compor o modelo de todas as classes.

Para o outono, foram obtidos modelos com altos coeficientes de determinação, de 0,$82 ; 0,83 ; 0,85$ e 0,82 para a umidade das classes $\mathrm{AA}, \mathrm{AB}, \mathrm{AC}$ e $\mathrm{B}$, respectivamente.

Tabela 4 - Modelos matemáticos ajustados para a estimativa da umidade de combustíveis florestais da fazenda experimental de Rio Negro - PR.

Table 4 - Mathematical models for estimation of fuel moisture of Rio Negro Forest Experimental Farm.

\begin{tabular}{|c|c|c|c|c|c|}
\hline Estação & Material & $\mathrm{R} 2$ & $\mathrm{~S}_{\mathrm{yx}}$ & $\mathrm{F}$ & Modelo \\
\hline \multirow{2}{*}{ Inverno } & AA & 0,59 & 11,70 & 20,85 & $\mathrm{U}_{\mathrm{A}=} 311,672-55,309 \mathrm{GF}+$ \\
\hline & $\mathrm{B}$ & 0,28 & 39,21 & 6,44 & $\mathrm{U}_{\mathrm{B}=} 88,875+1,609 \mathrm{U}$ \\
\hline \multirow{4}{*}{ Primavera } & AA & 0,64 & 20,95 & 13,69 & $\mathrm{U}_{\mathrm{A}=}-41,0643+1,5111 \mathrm{U}+1,7216 \mathrm{P}$ \\
\hline & $\mathrm{AB}$ & 0,76 & 26,16 & 23,18 & $\mathrm{U}_{\mathrm{AB}}=184,5024+1,4545 \mathrm{U}-8,0303 \mathrm{~F}+$ \\
\hline & $\mathrm{AC}$ & 0,79 & 14,67 & 27,54 & $\mathrm{U}_{\mathrm{AC}}=250,5632+0,9582 \mathrm{U}-4,8418 \mathrm{~F}+$ \\
\hline & $\mathrm{B}$ & 0,33 & 23,66 & 7,97 & $\mathrm{U}_{\mathrm{B}=} 230,3618-19,2836 \mathrm{GF}+$ \\
\hline \multirow{4}{*}{ Verão } & $\mathrm{AA}$ & 0,85 & 14,79 & 27,89 & $\mathrm{U}_{\mathrm{A}=} 97,79+0,69 \mathrm{U}+2,83 \mathrm{P}-25,55 \mathrm{GF}+$ \\
\hline & $\mathrm{AB}$ & 0,73 & 18,78 & 19,93 & $\mathrm{U}_{\mathrm{AB}=} 227,14-10,41 \mathrm{P}+33,54 \mathrm{GF}+$ \\
\hline & $\mathrm{AC}$ & 0,66 & 23,82 & 28,56 & $\mathrm{U}_{\mathrm{AC}}=335,64-32,10 \mathrm{GF}+$ \\
\hline & $\mathrm{B}$ & 0,60 & 23,39 & 11,68 & $\mathrm{U}_{\mathrm{B}=} 311,871-3,155 \mathrm{P}-34,019 \mathrm{GF}+$ \\
\hline \multirow{4}{*}{ Outono } & AA & 0,82 & 13,00 & 11,71 & $\mathrm{U}_{\mathrm{A}}=87,94+0,04 \mathrm{U}+1,40 \mathrm{P}-1,25 \mathrm{~F}++2,04 \mathrm{GF}+$ \\
\hline & $\mathrm{AB}$ & 0,85 & 23,68 & 20,15 & $\mathrm{U}_{\mathrm{AB}}=293,11+-0,30 \mathrm{U}-0,59 \mathrm{P}-3,03 \mathrm{~F}+$ \\
\hline & $\mathrm{AC}$ & 0,83 & 23,73 & 28,87 & $\mathrm{U}_{\mathrm{AC}}=424,66-5,85 \mathrm{P}-46,86 \mathrm{GF}+$ \\
\hline & $\mathrm{B}$ & 0,82 & 25,29 & 11,10 & $\mathrm{U}_{\mathrm{B}=} 185,62+1,18 \mathrm{U}-3,66 \mathrm{P}-3,31 \mathrm{~F}++10,57 \mathrm{GF}+$ \\
\hline
\end{tabular}

Cerne, Lavras, v. 18, n. 3, p. 371-376, jul./set. 2012 
De maneira geral, o índice de perigo de incêndios $\mathrm{FMA}^{+}$produziu melhores resultados no desenvolvimento das equações para estimar a umidade dos materiais combustíveis, principalmente para o período do outono.

\section{CONCLUSÕES}

Foi comprovada a hipótese de que os índices de perigo de incêndios FMA e $\mathrm{FMA}^{+}$apresentaram boa correlação com o material combustível, sendo viável a estimativa da umidade dos combustíveis finos por meio desses índices.

A $\mathrm{FMA}^{+}$, quando comparada com a FMA, apresentou melhores resultados e foi a variável de maior importância nos modelos de estimativa da umidade dos materiais combustíveis.

$\mathrm{O}$ uso do índice $\mathrm{FMA}^{+}$, para estimativa da umidade dos materiais combustíveis, produz informações de qualidade, com rapidez e eficiência.

\section{REFERÊNCIAS}

ALVES, M. G. A.; BATISTA, A. C.; SOARES, R. V.; OTAVIANO, M.; MARCHETTI, M. Fuel moisture sampling and modeling in Pinus elliottii Engelm: plantations based on weather conditions in Paraná, Brazil. Biogeosciences and Forestry, Bologna, n. 489, p. 99-103, 2009.

BATISTA, A. C. Avaliação da queima controlada em povoamentos de Pinus taeda L. no norte do Paraná. 1995. 108 p. Tese (Doutorado em Engenharia Florestal) Universidade Federal do Paraná, Curitiba, 1995.

BROWN, J. K.; OBERHEU, R. D.; JOHNSTON, C. M. Handbook for inventorying surface fuels and biomass in the Interior West. Odgen. Oxford: Intermountain Forest and Range Experiment Station, 1982. 48 p. (General Technical Report INT, 129).

FOSBERG, M. A.; LANCASTER, J. W.; SCHROEDER, M. J. Fuel moisture response: drying relationships under standar and field conditions. Forest Science, Bethesda, v. 16, p. 121-128, 1970.

NUNES, J. R. S. FMA ${ }^{+}$: um novo índice de perigo de incêndios florestais para o estado do Paraná, Brasil. 2005. 150 p. Tese (Doutorado em Engenharia Florestal) - Universidade Federal do Paraná, Curitiba, 2005.

NUNES, J. R. S. Sistema integrado de controle de incêndios florestais para o estado do Paraná. Curitiba: UFPR, 2007. $130 \mathrm{p}$.

SOARES, R. V. Determinação de um índice de perigo de incêndios para a região centro paranaense, Brasil. 1972. 72 f. Dissertação (Mestrado) - CATIE/IICA, Turrialba, 1972.

SOARES, R. V.; BATISTA, A. C. Incêndios florestais: controle, efeitos e uso do fogo. Curitiba: [s.n.], 2007. 250 p.

SOARES, R. V.; BATISTA, A. C.; NUNES, J. R. S. Incêndios florestais no Brasil: o estado da arte. Curitiba: [s.n.], 2009. $240 \mathrm{p}$.

YEBRA, M.; CHUVIECO, E.; RIAÑO, D. Investigation of a method to estimate live fuel moisture content from satellite measurements in fire risk assessment. Forest Ecology and Management, Amsterdam, v. 234, n. 1, p. S32, 2006. Supplement.

Cerne, Lavras, v. 18, n. 3, p. 371-376, jul./set. 2012 